\title{
ENCUESTA SOBRE IMPLEMENTACIÓN DE INVESTIGACIÓN EN CENTROS EDUCACIONALES EN CHILE: CUMPLIMIENTOS DE ASPECTOS REGULATORIOS ÉTICOS Y LEGALES
}

\author{
Catalina Merino-Osorio ${ }^{1}$, Gustavo Mattar T. ${ }^{1}$, Jaime Leppe Z. ${ }^{1}$, Paz Bahamondes M. ${ }^{1}$, Juan Alberto \\ Lecaros $^{2}$
}

\begin{abstract}
Resumen: Antecedentes: La investigación en centros educacionales es una fuente frecuente de estudios de las áreas sociales y salud. Objetivo: Determinar el conocimiento y la adherencia a la regulación ética y legal vigente de la investigación realizadas con niños en centros educacionales. Metodología: Estudio transversal tipo encuesta online. Se consultó sobre el nivel de conocimiento y adherencia a la regulación ética y legislación vigente en Chile, para realizar investigación con menores de edad. Resultados: 126 centros respondieron la encuesta; $69 \%$ de zonas urbanas; $60 \%$ son centros municipales y $34 \%$ particulares subvencionados. En un 31,8\% de los centros se realizó investigación, $41 \%$ de salud y 30,8\% de educación. El 27,5\% informó que la investigación contaba con aprobación del comité ético-científico para su implementación, un $82 \%$ señaló no recibir ni tener conocimiento de los aspectos regulatorios sobre la investigación con seres humanos. Conclusión: Existe bajo conocimiento y adherencia a la regulación ética y legal de la investigación con niños en centros educacionales. Se realizó una jornada de difusión de los resultados y se propuso un flujograma generado por expertos orientado a los directores de centros educacionales para contribuir a la adherencia de los aspectos éticos y regulatorios de la investigación en centros educacionales.
\end{abstract}

Palabras clave: bioética, investigación, centros educacionales, jurisprudencia, educación.

Survey research implementation in educational centers in Chile: fulfillments of ethical and legal regulatory aspects

Abstract: Background: Research in educational centers is a frequent source of social and health related studies. Objective: To determine the knowledge and adherence to the current legal and ethical regulations to research performed on children at educational institutions. Methodology: Cross-sectional study online survey-type. The level of knowledge and adherence to the ethical regulation and legislation in Chile, to conduct research with minors was consulted. Results: 126 educational institutions responded the survey; $69 \%$ belonged to urban centers; $60 \%$ corresponded to those under the Municipality umbrella and 34\% to charter schools. Research was performed in 31,8\% of the consulted institutions, $41 \%$ of the research was on health issues and $30,8 \%$ on education. The $27,5 \%$ answered that they had the approval of ethical-scientific committee, $82 \%$ answered that they neither received nor had any awareness of regulatory aspects on research. Conclusion: There is low knowledge and adherence to ethical and legal regulation to research performed with children in educational centers. A journey for dissemination of results was performed and a flow chart was created by experts and proposed to the school directors to contribute to the adherence of ethical and regulatory aspects of research in educational centers.

Key words: bioethics, research, educational centers, jurisprudence, education

Enquete sobre a realizaçáo de pesquisas em centros educacionais no Chile: cumprimentos de aspectos regulatórios éticos e legais

Resumo: Antecedentes: A pesquisa em centros educacionais é uma fonte frequente de estudos das áreas sociais e de saúde. Objetivo: determinar o conhecimento e a adesão à regulamentação ética e legal vigente de pesquisa realizada com crianças em centros educacionais. Metodologia: estudo transversal de tipo enquete on-line. Consultou-se sobre o nível de conhecimento e adesão ao regulamento ético e a legislação vigente no Chile, para realizar a pesquisa com menores de idade. Resultados: 126 centros responderam à enquete; $69 \%$ em áreas urbanas; $60 \%$ são centros municipais e $34 \%$ centros particulares subsidiados. Em 31,8\% dos centros foram realizadas pesquisas, $41 \%$ de saúde e 30,8\% de educação. 27,5\% relataram que a pesquisa teve aprovação do Comitê de etico-cientifico para a sua execução, $82 \%$ disseram náo receber ou ter conhecimento dos aspectos regulatórios da pesquisa com seres humanos. Conclusão: Existe pouco conhecimento e adesão ao regulamento ético e legal de pesquisa envolvendo crianças em centros educacionais. Fora realizada uma conferência para a divulgação dos resultados e fora proposto um fluxograma gerado por especialistas, que visam os diretores de centros educacionais para contribuir para a aderência dos aspectos éticos e regulatórios de pesquisa em centros educacionais.

Palavras-chave: bioética, pesquisa, centros educacionais, jurisprudência, educação

\footnotetext{
${ }^{1}$ Carrera de Kinesiología, Facultad de Medicina Clínica Alemana Universidad del Desarrollo, Chile Correspondencia: catalinamerino@udd.cl

${ }^{2}$ Observatorio de Bioética y Derecho, Universidad del Desarrollo, Chile
} 


\section{Introducción}

Investigación es todo proceso que, mediante la aplicación del método científico, procura obtener información relevante y fidedigna para entender, verificar, corregir o aplicar conocimiento(1). Según la OMS, la investigación con seres humanos hace referencia a "cualquiera actividad de ciencias sociales, biomédica, conductual o epidemiológica que implique la recopilación o el análisis sistemáticos de datos con el objetivo de generar nuevos conocimientos y en la que seres humanos: 1) estén expuestos a la manipulación, la intervención, la observación u otra interacción con los investigadores, directamente o mediante la alteración de su entorno; o 2) puedan ser identificables individualmente mediante la obtención, la preparación o el uso por parte de los investigadores de materiales biológicos o registros médicos o de otro tipo"(2).

Después de la Segunda Guerra Mundial, como consecuencia de las violaciones a los derechos humanos que sufrieron miles de personas que fueron sometidas a experimentos médicos por el Régimen Nazi, se crearon normas éticas de investigación internacionales como el Código de Nüremberg y luego la Declaración de Helsinki(3). Esta última hizo exigible la revisión previa de la investigación médica por comités de ética, cuya función es proteger el bienestar y los derechos de las personas que participan como sujetos en una investigación biomédica(4). Hoy se exige que no tan solo la investigación deba conducirse respetando los principios y responsabilidades que establece la Declaración de Singapur sobre la Integridad en la Investigación(5), sino que también, cuando esta involucra a seres humanos, deba conducirse respetando los principios y normas de la pautas éticas internacionales de investigación con seres humanos, tales como, por ejemplo, la Declaración de Helsinki, las Pautas CIOMS(6), las Normas Éticas de Investigación de la OPS(7), entre otras.

La investigación con niños en centros educacionales tiene una larga data. Existe registro de que ya en el siglo XIX era practicada por inspectores de escuelas, quienes emitían sus resultados en los informes administrativos(8). Lo característico de estas investigaciones es que se cuenta con poblaciones cautivas de un amplio rango de edad que abarca desde niños a jóvenes. Pero en el siglo pasado ha habido una creciente sensibilidad de que estas poblaciones, por su minoría de edad, sean consideradas grupos vulnerables que requiere una especial protección. Con todo, desde la adopción de la Convención sobre de los Derechos del Niño de Naciones Unidas (1989)(9) se ha promovido un enfoque que comprende a los niños no tan solo como sujetos de protección, sino también como sujetos de derechos que deben ser asistidos en el ejercicio de sus derechos personalísimos y que deben ser respetados en sus decisiones en función de su edad y madurez.

Uno de los principios básicos de la ética de la investigación afirma que todo ser humano debe ser respetado en su decisión de participar o negarse a participar de una investigación, y ello mediante un proceso de consentimiento previo, libre e informado. En este sentido, se considera a los sujetos de investigación como coparticipantes durante el proceso de investigación $(10,11)$. Son los investigadores quienes tienen la obligación de que los sujetos enrolados no sean forzados, manipulados o presionados indebidamente para participar en la investigación(12). El consentimiento informado es el proceso que garantiza esta obligación, el cual se inicia antes de la ejecución del estudio y comprende la explicación y firma del documento que resguarda dicho proceso. El consentimiento informado se puede definir como "la decisión de participar en la investigación, adoptada por una persona competente que ha sido informada convenientemente, que ha comprendido adecuadamente la información, y que, después de considerarla, ha adoptado esa decisión sin haber sido sometida a coacción, influencia o incentivos indebidos, o intimidación" (13).

Cuando las investigaciones involucran niños, las pautas éticas internacionales recomiendan autorizar la participación de personas que pertenecen a este colectivo bajo las siguientes condiciones: 1) cuando se justifica investigar en ellas porque no podría realizarse con eficacia comparable en personas capaces; 2) existe un beneficio directo para la salud de los participantes y si no es así, al menos, se investiga en problemas de salud de los niños; y, por último, 3) si no hay beneficio directo para los nińos que participan en la investigación, que estos estén sometidos a riesgos mínimos(14). 
Otras de las condiciones imprescindibles se relacionan con la voluntariedad de participar, ya que, tratándose de personas que no son competentes para consentir informadamente si participan o no en una investigación, debido a su minoría de edad, se requiere que sus representantes, usualmente sus padres, consientan por ellos.

Lo anterior no significa que los niños no deban ser tenidos en cuenta en la decisión de participar o no en una investigación. El estándar general de las pautas éticas internacionales recomienda que los niños sean respetados como personas, teniendo en cuenta su edad y madurez, mediante el proceso de asentimiento, con el cual se busca adscribir al niño en la investigación en la mayor medida posible. Lo mínimo que se debe respetar, cuando se buscar incluir niños en una investigación, es su derecho a disentir, esto es, el derecho a expresar su voluntad de no participar antes del inicio o en cualquier momento durante la ejecución de la investigación. El proceso de asentimiento es coherente con el derecho reconocido en la Convención sobre los Derechos del Niño en su artículo 12 que dispone: "Los Estados garantizarán al niño que esté en condiciones de formarse un juicio propio, el derecho de expresar su opinión libremente en todos los asuntos que le afecten, teniéndose debidamente en cuenta sus opiniones, en función de su edad y madurez." (15-17).

En la doctrina y práctica de la investigación actual, buscando el máximo respeto del derecho a la información y decisión de los menores en investigación, se ha desarrollado el concepto de "consentimiento parental pasivo", que consiste en que los padres solo expresen su voluntad de que el menor maduro no participe, siendo este quien otorgue su consentimiento, habiendo sido previamente informado. Este tipo de consentimiento tiene sentido para investigaciones en el área de la ciencias sociales/educacionales, en razón de que el consentimiento directo de los padres puede afectar a los mismos objetivos de la investigación (v. gr., confidencialidad de los datos de los menores en temas sensibles, como sexualidad, uso de drogas, etc.), vale decir, hay razones fundadas para no involucrar a los padres si los menores son capaces de entender dichos objetivos y de consentir (menores maduros), y la investigación va en beneficio del colectivo de los menores y tiene riesgos míni- mos(18). Sin duda, en la práctica, el uso de esta herramienta requiere de una adecuada ponderación de los beneficios y riesgos para los menores maduros por parte del comité ético científico.

En relación con el proceso ético de la investigación en centros educacionales, algunos estudios han reportado que se destina escaso tiempo para explicar el propósito de la investigación y, pese a esto, se sigue adelante con ella(19). Otros estudios ponen en evidencia casos en que es el director del establecimiento quien otorga el consentimiento en nombre de sus estudiantes, cuestión que atenta contra los estándares éticos internacionales en investigación $(20,21)$. Otro estudio reportó que más de la mitad (55\%) de los profesores miembros de una oficina de investigación médica de educación en Estados Unidos desconocían si su institución tenía políticas que salvaguardaran a la integridad y el bienestar de los estudiantes que participan en investigación(17). En Chile, la Comisión Nacional de Investigación Científica y Tecnológica en Chile (CONICYT) realizó una evaluación ética de los proyectos financiados durante 2012, el cual reveló que alrededor de un $40 \%$ de los proyectos señalaba explícitamente que se adhería a normas éticas locales e internacionales, y que contaba con un documento de consentimiento informado; no obstante, solo el $62,1 \%$ de los proyectos financiados por el Fondo Nacional de Desarrollo Científico y Tecnológico (FONDECYT) y el $63,6 \%$ de los financiados por el Fondo Nacional de Investigación y Desarrollo en Salud (FONIS) informaban que el participante o su representante legal habían recibido suficiente información respecto del estudio y que esta se había entendido completamente(22).

Las normas legales que regulan la investigación con seres humanos en Chile están recogidas en la ley $\mathrm{N}^{\circ} 20.120$, promulgada en 2006, complementadas y desarrolladas en su reglamento, el Decreto $\mathrm{N}^{\circ} 114$ del Ministerio de Salud, promulgado en 2011 y modificado en 2013. El reglamento define la investigación con seres humanos en un sentido bastante lato, de modo que no la reduce a las investigaciones biomédicas que implican intervención física o psíquica, sino que comprende a cualquier investigación que implique interacción con seres humanos, incluyendo el uso de datos sensibles. Se debe entender entonces que las 
investigaciones en ciencias sociales/educacionales, que implican interacción con seres humanos y/o usos de datos sensibles, están sujetas a esta normativa legal.

Si bien esta ley y su reglamento establecen garantías y derechos mínimos, como por ejemplo el consentimiento informado del participante o su representante legal, que la investigación sea realizada por profesionales idóneos, que cuente con la autorización del director del establecimiento donde se efectúa, previa aprobación de un comité ético científico, contiene exigencias formales que dificultan la investigación, el artículo 11 de la ley exige que el director del establecimiento debe firmar cada documento de consentimiento informado, actuando en calidad de ministro de fe, aunque puede delegar estas facultades. Esta formalidad es innecesaria para la investigación biomédica que implica mayores riegos como los ensayos clínicos con fármacos o dispositivos médicos, por lo que resulta aún más innecesaria para investigaciones en ciencias sociales como las que se realizan en centros educacionales. Sin duda, estas deficiencias de técnica legislativa hacen necesaria una modificación de nuestra legislación de investigación biomédica, de tal modo que exista un marco legal eficaz y comprensivo de toda investigación con seres humanos.

Respecto de la participación de los menores en las investigaciones, nuestra normativa legal se reduce a consagrar el derecho a expresar el disentimiento del menor para ingresar en un estudio o retirarse de él en cualquier momento. Aunque la versión anterior de dicho reglamento, la de 2011, consagraba un derecho de asentimiento de los menores maduros en estos términos: "los niños menores de 18 y mayores de 12 se permitirá, en la medida de lo posible, su presencia al acto para conocer su opinión", el reconocimiento que hace el reglamento vigente es sin duda insuficiente para respetar los derechos reconocidos en la Convención sobre los Derechos del Nińo, tratado ratificado y vigente para nuestro país, el cual en su artículo 12 garantiza a los menores que estén en condiciones de formarse una opinión propia el derecho a expresar su opinión libremente y que esta sea tenida en cuenta en función de su edad y madurez. Tanto las pautas éticas internacionales sobre investigación con seres humanos como el derecho com- parado reconocen el derecho de los menores de asentir participar en las investigaciones, debiendo recibir la información adecuada y suficiente en función de su edad y madurez.

\section{Metodología}

El objetivo del estudio es describir el proceso de investigación en centros educacionales, determinando el conocimiento, adherencia y respeto a la regulación ética y legal vigente, y las características de las investigaciones realizadas en centros educacionales.

Metodológicamente corresponde a un estudio descriptivo transversal, tipo encuesta online, dirigido a los 12061 establecimientos educacionales reconocidos por el Ministerio de Educación (MINEDUC) al año 2014 (identificados en la página web: "Más información, mejor educación" http://www.mime.mineduc.cl), independiente de su dependencia administrativa y constituyendo por tanto un muestreo de tipo censal.

Mediante una encuesta de 13 preguntas, cuyo formulario fue enviado por correo electrónico a los directores de dichos establecimientos, se identificó las dimensiones de conocimiento, respeto de la legislación y tipo de investigación realizada en los centros educacionales. Se invitó a participar del estudio a los directores de los establecimientos, previa autorización mediante consentimiento informado online. El formulario para contestar la encuesta estuvo disponible durante 22 días, enviándose un recordatorio previo al cierre.

Para el análisis de los datos se utilizó estadística descriptiva, utilizando el paquete estadístico STATA $12.0^{\circ}$.

Este estudio contó con la autorización del Comité Ética-Científico (CEC) de la Facultad de Medicina Clínica Alemana Universidad del Desarrollo, Acta No PG_02/2015.

\section{Resultados}

Se incluyó en el análisis un total de 125 encuestas (figura 1), de las cuales el 69\% ( $n=86)$ pertenecía a centros educacionales de zona urbana y el 31\% $(\mathrm{n}=39)$ a la zona rural. La principal dependencia administrativa de los centros fue un $60 \%$ de 
centros educacionales municipales y un $33,6 \%$ de establecimientos particulares subvencionados (tabla 1).

Figura 1: Diagrama descriptivo del proceso de selección de los participantes.

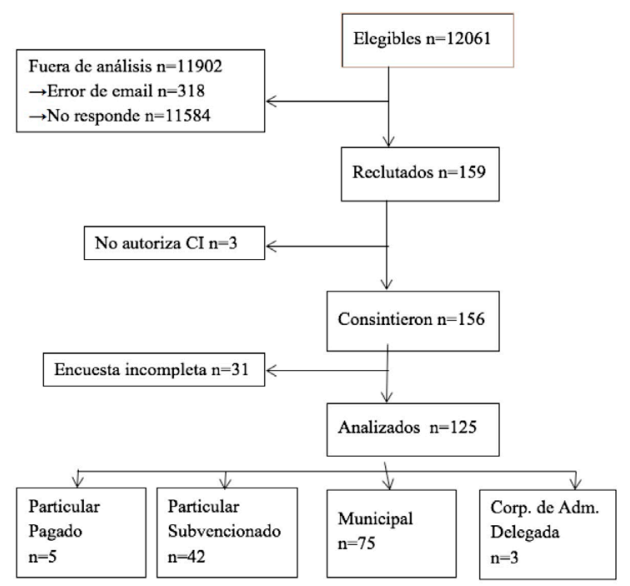

Tabla 1: Caracterización de centros educacionales según ubicación geográfica y dependencia administrativa $(\mathrm{n}=125)$

\begin{tabular}{|c|c|}
\hline \begin{tabular}{ll}
\multicolumn{2}{l}{ Ubicación geográfica } \\
$-\quad$ Rural \\
$-\quad$ Urbano
\end{tabular} & $\begin{array}{l}39(31 \%) \\
86(69 \%)\end{array}$ \\
\hline \begin{tabular}{ll}
\multicolumn{2}{l}{ Dependencia administrativa } \\
- & Municipal \\
- & Particular pagado \\
- & Particular subvencionado \\
- & Corporación de administración \\
& delegada
\end{tabular} & $\begin{array}{l}75(60 \%) \\
5(4 \%) \\
42(33,6 \%) \\
3(2,4 \%)\end{array}$ \\
\hline
\end{tabular}

De los centros analizados según ubicación geográfica, el 31\% ( $n=39)$ reportó haber realizado investigación durante 2014, principalmente en zonas urbanas $(69 \%)$ y en centros de dependencia municipal $(n=10)$, en la zona rural, y en centros particulares subvencionados $(\mathrm{n}=12)$, en la zona urbana. Las áreas disciplinares de investigación más

Tabla 2: Caracterización de los centros educacionales, área disciplinar y aprobación por CEC de la investigación realizada en el año 2014, según ubicación geográfica $(n=39)$.

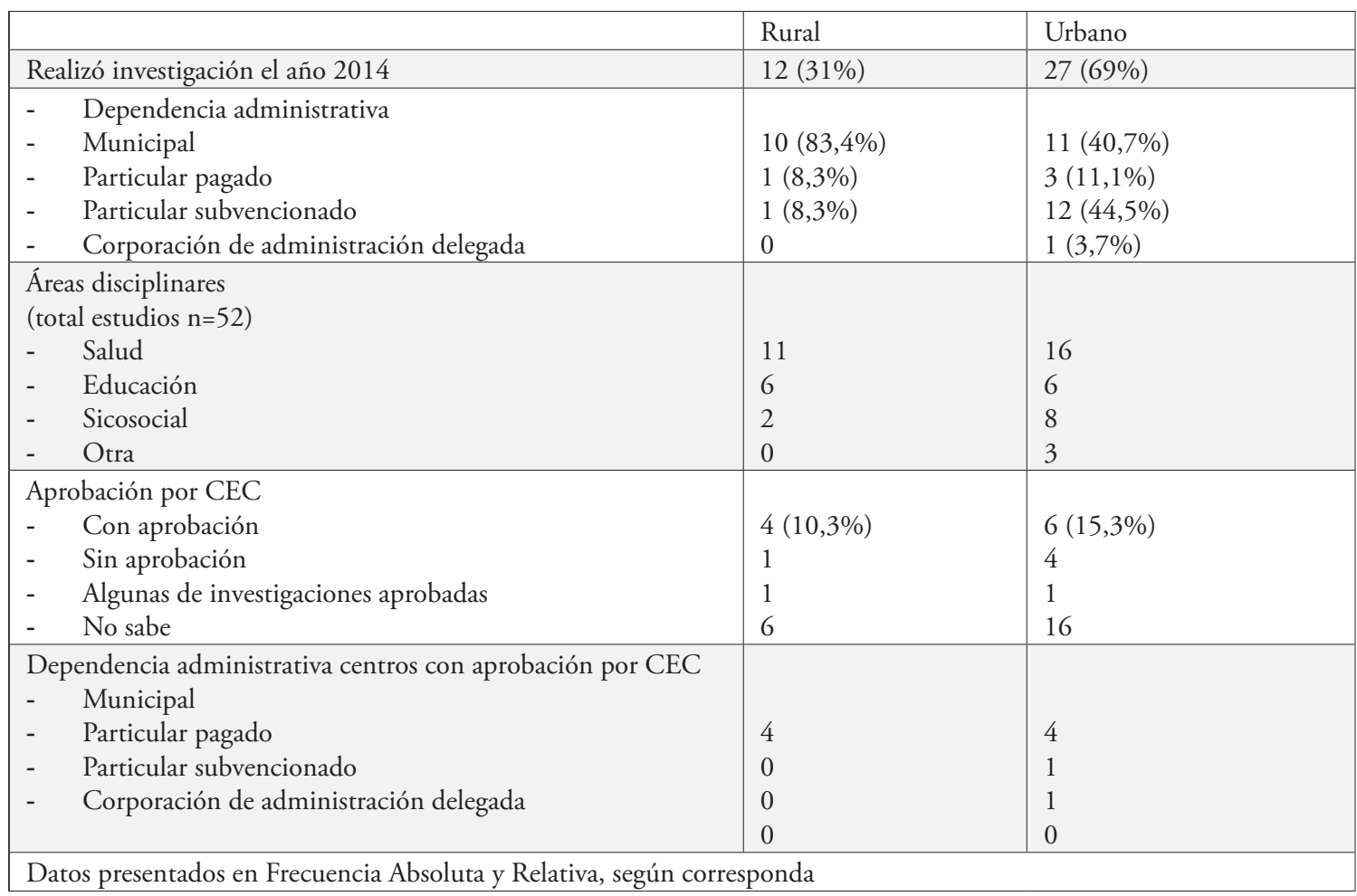


frecuentes fueron salud (52\%), educación (23\%), sicosocial $(19,2 \%)$ y otras ciencias $(5,8 \%)$, distribuidas en los 39 centros educacionales que informaron un total de 52 estudios. El 25,6\% ( $\mathrm{n}=10)$ de estos centros aseguró que las investigaciones contaban con aprobación por parte de un CEC, correspondiendo estos a centros principalmente de zona urbana y de dependencia municipal (tabla 2).

De los 39 centros que aseguraron haber realizado investigación durante 2014, 13\% de ellos reportó que estas investigaciones no contaban con aprobación de CEC, un 56,4\% no sabía si las investigaciones realizadas en sus centros habían contado con dicha aprobación y el 5\% restante aseguraba que algunas de ellas tenían aprobación de CEC (figura 2).

Figura 2: Adherencia de los centros educacionales a la aprobación de las investigaciones realizadas el año 2014 por un comité ético científico (CEC) $(\mathrm{n}=39)$

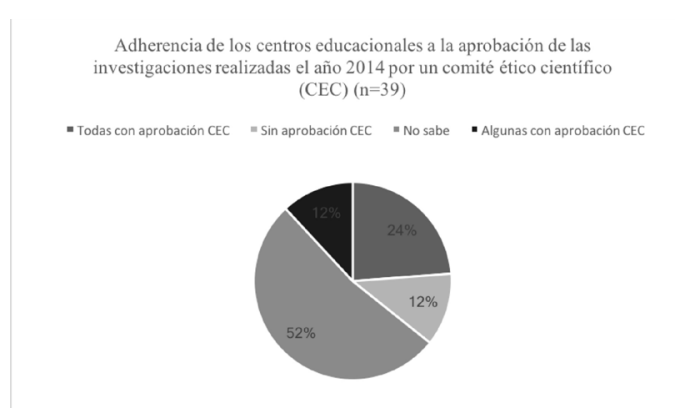

El 69,2\% de los centros reporta que en las investigaciones realizadas en 2014 se utilizó consentimiento informado y el 59\% aseguró haber utilizado asentimiento informado en los sujetos menores de edad.

Respecto del conocimiento sobre la regulación ética y legal de la investigación en seres humanos, el $82 \%$ de los centros aseguró no haber recibido información alguna por parte de las autoridades de educación, ni estar en conocimiento de que toda investigación realizada con seres humanos debe estar aprobada por un CEC. Por otra parte, del total de centros educacionales donde no se realizó investigación en 2014 ( $\mathrm{n}=86)$, el 50\% aseguró que la autorización para participar en el estudio debe solicitarse solo al director del establecimiento, el $15 \%$ señaló que solo al apoderado, y el $35 \%$ que debía solicitarse al alumno y al apoderado. Finalmente, el $24,8 \%$ del total de directores que participó del estudio reportó estar en desconocimiento que, una vez concluida la investigación, los resultados deben ser entregados a la institución y a cada uno de los participantes, ya sea por compromiso de los investigadores o porque el participante lo solicitó.

\section{Discusión}

Alrededor de un tercio de los directores de centros educacionales encuestados reportaron haber realizado investigación durante 2014. Estas se desarrollaron principalmente en zonas urbanas y en centros educacionales municipales. La diferencia en la proporción del tipo de dependencia del establecimiento en el cual se realizó mayor cantidad de investigación en 2014, identificado por zona geográfica, podría estar dado por la mayor proporción de centros municipales en zona rural $y$ particulares subvencionados en zona urbana, $y$, por tanto, un mayor acceso a estas poblaciones.

Respecto de la adherencia de los directores a la regulación ética y legal, el 56,4\% de los directores de centros educacionales afirmó desconocer si las investigaciones realizadas en sus centros habían contado con la aprobación de un CEC. Este dato deja en evidencia que existe una brecha entre el conocimiento de la regulación ética y legal de la investigación en seres humanos y el cumplimiento por parte de los directores de establecimientos educacionales de las exigencias de dicha regulación. Esto se sustenta en que el $82 \%$ de los centros aseguró no haber recibido información alguna por parte de las autoridades de educación, ni estar en conocimiento de que toda investigación realizada con seres humanos debe estar aprobada por un CEC $(24,8 \%)$. Lo anterior pudiera explicar la falta de resguardos de los directores de centros educacionales en la protección de los menores de edad pertenecientes a su institución involucrados en investigación. Con todo, esto no justifica ni aminora la responsabilidad pública de la cual las autoridades educacionales en Chile deben hacerse cargo. Como representantes del Estado, ellas deben seguir las recomendaciones de la ONU y respetar y promover los derechos establecidos 
en tratados vigentes, como la Convención sobre los Derechos del Nińo, ya que estos instrumentos internacionales estipulan que los responsables gubernamentales en la toma de decisiones en educación son quienes deben promover el respeto de los derechos del niño y otorgar de la información necesaria para proteger a este colectivo vulnerable(15).

Los resultados del presente estudio arrojaron una desigualdad entre la proporción de estudios que utilizaron consentimiento informado (69,2\%) versus los que utilizaron asentimiento informado (59\%), lo cual lleva a reflexionar respecto de la responsabilidad de los investigadores que realizan estudios con seres humanos en centros educacionales, quienes, al parecer, descuidan el rigor metodológico y el estándar ético y legal exigible en la investigación, al no considerar, a cabalidad y en integridad en sus investigaciones, el principio de autonomía de los participantes. La solicitud de asentimiento informado en los menores de edad es una obligación ética y legal que no se ve representada en los resultados de esta encuesta. Sería interesante identificar en futuros estudios si esto se debe a la falta de conocimiento por parte de las investigadores de la regulación ética y legal vigente, o si se debe a una negligencia en el cumplimiento del rigor ético y metodológico de la investigación. Por ello, se hace necesaria la formación bioética de los investigadores acerca de la importancia y relevancia de la investigación con seres humanos en centros educacionales, en donde la aplicación de pautas éticas internacionales pudiera ser una estrategia de educación de la comunidad científica(23).

La principal limitación de este estudio se basa en la baja tasa de respuesta, debido a la cantidad de correos rebotados y a aquellos que, ingresados como oficiales según el MINEDUC, no permitían acceder directamente al director del establecimiento. Tal situación revela una falta de eficiencia en los procesos de comunicación, que plantea la necesidad de actualización del medio de contacto entre autoridades de gobierno y de centros educacionales, y el aseguramiento en la recepción de comunicados relevantes. Esta situación dificulta la capacidad de difusión de la información desde las autoridades a los directores de establecimientos educacionales, lo cual podría explicar, en par- te, que los encuestados reporten no haber recibido información acerca de la regulación ética y legal de la investigación en centros educacionales o, simplemente, podría deberse a la simple ausencia de comunicación con información de este tipo.

Se concluye entonces que, a pesar de existir un marco legal que rige el proceso de investigación con seres humanos en centros educacionales y pautas éticas de investigación con seres humanos conocidas en la comunidad internacional de la investigación, existen falencias en la implementación de los requisitos éticos y legales que regulan las investigaciones en centros educacionales, debido principalmente al desconocimiento, en primer lugar, y luego a la falta de adherencia a esta regulación. Esta situación expone a los menores de edad en centros de educación sometidos a investigación a un estado de vulnerabilidad, lo cual constituye una señal de alarma para las autoridades de educación, directores de centros de educación, para los padres y/o apoderados y para los mismos estudiantes.

Es necesario generar políticas de educación y estrategias de difusión de estas políticas que permitan aumentar el conocimiento y adherencia a los aspectos éticos y legales que regulan la investigación en centros educacionales. Asimismo, incorporar a investigadores y autoridades como parte del proceso de mejora de estas políticas y programas de difusión, y con ello contribuir a la protección de los sujetos de estudio y a la ejecución de proyectos de investigación bajo el rigor ético y metodológico que requiere la ciencia en nuestro país en el concierto internacional. 
Encuesta sobre implementación de investigación en centros educacionales en Chile - Catalina Merino-Osorio et al.

\section{Referencias}

1. Simons H, Usher R. Situated Ethics in Educational Research. $1^{\text {th. }}$ Ed. New York: Routledge; 2007.

2. Organización Panamericana de la Salud. Pautas y orientación operativa para la revisión ética de la investigación en salud con seres humanos. Washington, DC: OPS, 2012: 42. Available from: URL: http://www.paho.org/hq/index. php?option=com_docman\&task=doc_view\&gid=22738\&Itemid=

3. Fluss S. How the Declaration of Helsinki developed? Bioética Informa 2000; VI (19): 13-17.

4. León F. Ética y responsabilidad social de las instituciones de salud en Chile. Santiago de Chile: Ediciones Centro de Políticas Públicas Universidad Católica. 2012.

5. Declaración de Singapur sobre la Integridad en la Investigación. 2010; 1(1). Available from: http://www.singaporestatement.org/Translations/SS_Spanish.pdf.

6. Council for International Organizations of Medical Sciences. International Ethical Guidelines for Biomedical Research Involving Human Subjects. Geneva: CIOMS; 2002: Preamble.

7. Gracia D. Investigación en sujetos humanos: Implicancias lógicas, históricas y éticas. En Pellegrini A, Macklin R. (eds.) Investigación en sujetos humanos. Experiencia Internacional. Santiago: OPS/OMS; 1999: 63-81.

8. Brunner J, Salazar F. La investigación educacional en Chile: Una aproximación bibliométrica no convencional. Documento de Trabajo CPCE No 1. Santiago de Chile: Centro de políticas comparadas de Educación; 2009,

9. Asamblea General Naciones Unidas. Convención sobre los derechos del niño. 20 de noviembre de 1989. Madrid: Rex Media; 2006.

10. Alderson P. As crianças como pesquisadoras: Os efeitos dos direitos de participaçao sobre a metodología de pesquisa. Educ Soc Campinas 2005; 26(91): 419-442.

11. Standish P. Data return: The place of the given in educational research. Journal of philosophy of Education 2001; 35(3).

12. McNamee M. Introduction: Whose ethics, which research? Journal of philosophy of Education 2001; 35(3).

13. Organización Panamericana de la Salud. Pautas y orientación operativa para la revisión ética de la investigación en salud con seres humanos. Washington, DC: OPS; 2012: 41.

14. Asamblea general Naciones Unidas. Convención sobre los Derechos del Niño, 3 de noviembre de 2010. España: Rex Media; 2010.

15. Tickle L. Opening windows, closing doors: Ethical dilemmas in educational action research. Journal of Philosophy of Education 2001; 35(1): 345-359.

16. 13. León C. Información y consentimiento informado de menores de edad en Chile. Revista Chilena de Pediatría 2012; 83(2): 113-116.

17. Department of Health and Human Services, National Institutes of Health, Office of Protection from Research Risks. Title 21: Public Welfare. Part 56: Institutional Review Boards. Washington, DC: 7 DHHS; 1991.

18. Chartier M, Vander Stoep Ann, et al. Passive Versus Active Parental Consent: Implications for the Ability of Schoolbased Depression Screening to Reach Youth at Risk. J Sch Health 2008; 78(3): 157-186.

19. Howe K, Moses M. The Ethics of Educational Research. American Educational Research Association 1999; 24: 21-29.

20. Heath S, Charles V, Crow G, Wiles R. Informed consent, gatekeepers and go -betweens: negotiating consent in childand youth-orientated institutions. British Educational Research Journal 2007; 33(3).

21. Mavis B, Henry R. Being uninformed on informed consent: a pilot survey of medical education faculty. BMC Med Educ 2005; 5(12).

22. Castañeda M, Huspenina V, Montaño S, Quintero R. Evaluación ética de proyectos de investigación financiados por la Comisión Nacional de Investigación Científica y Tecnológica, Chile 2012. Bioética y Derecho 2014; 31: 38-50.

23. Rodríguez E. Comités de evaluación ética y científica para la investigación en seres humanos y las pautas CIOMS 2002. Acta Bioethica 2004; 10(1).

Recibido: 9 de febrero de 2016

Aceptado: 6 de abril de 2016 